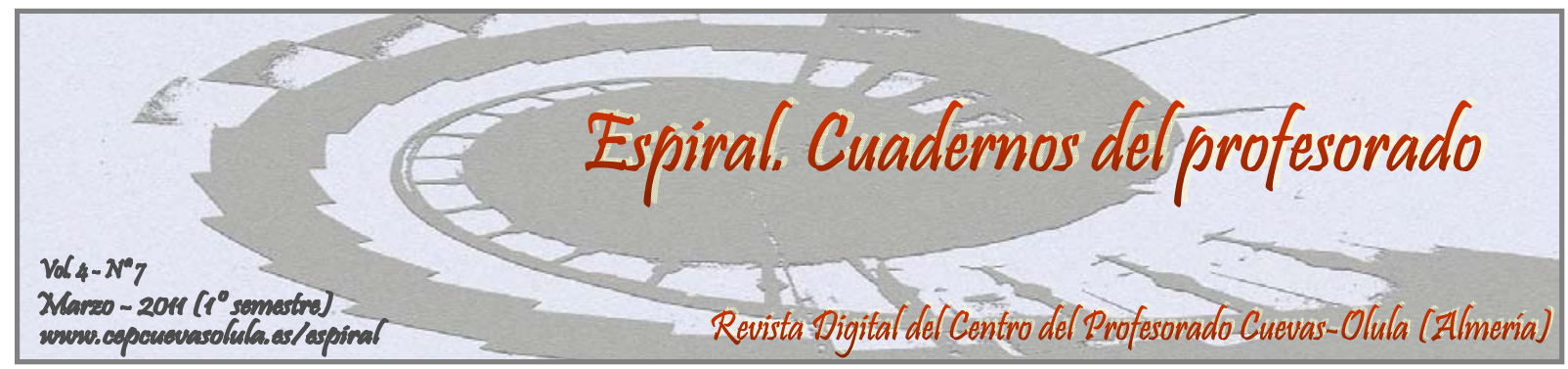

\title{
VIDEOJUEGOS ACTIVOS, VIDEOJUEGOS CONVENCIONALES Y ACTIVIDAD FÍSICA EN ADOLESCENTES DE SECUNDARIA
}

\section{ACTIVE VIDEO GAMES, CONVENTION VIDEO GAMES AND ADOLESCENT PHYSICAL ACTIVITY IN SECONDARY}

\author{
Juan Ignacio Beltrán Carrillo
}

\author{
IES Mar Serena de Pulpí, Almería, España
}

RESUMEN: El objetivo del presente trabajo es conocer el tiempo dedicado por una muestra de 570 adolescentes de secundaria a los videojuegos activos, los videojuegos convencionales y la práctica de actividad física de intensidad moderada-vigorosa. Las pruebas t para muestras independientes realizadas indicaron que los chicos dedican más tiempo que las chicas a la práctica de actividad física y al uso de videojuegos convencionales y activos. Los análisis de correlación lineal simple efectuados señalaron que en las chicas estas conductas no presentan relación alguna, mientras que en el grupo de chicos, los que más juegan a videojuegos activos son aquellos que más juegan a videojuegos convencionales y más tiempo dedican a la actividad física. Estos datos añaden una información interesante a la línea de investigación que estudia las relaciones entre el uso de medios tecnológicos y los estilos de vida activo y sedentario.

Palabras clave: estilo de vida activo, medios tecnológicos, conductas sedentarias.

ABSTRACT: This paper examines the time spent in active videogames, sedentary videogames and moderate-vigorous physical activity by a sample of 570 secondary school students. According to the between-subjects t tests, boys spend more time than girls in physical activity and both active and sedentary videogames. On the other hand, correlation analysis showed these behaviours are independent among girls while, in the male group, youngsters who play active videogames in a bigger extent are those who spend more time in sedentary videogames and are more physically active. These data add interesting information to the research line which studies in depth relationships between screen media usage and active-sedentary lifestyle.

Key words: active lifestyle, screen media, sedentary behaviours.

Beltrán Carrillo, J.I. (2011). Videojuegos activos, videojuegos convencionales y actividad física en adolescentes de secundaria. Espiral. Cuadernos del Profesorado [en línea], 4(7), 23-27. Disponible en: http://www.cepcuevasolula.es/espiral.

Fecha de recepción: 06/12/2010

Fecha de aceptación: 08/02/2011
Enviar correspondencia a: nachobeltranlorca@hotmail.com

\section{Agradecimientos.}

Los datos para la publicación de este artículo han sido extraídos del proyecto: Videojuegos activos, medios tecnológicos sedentarios y actividad física: un estudio con adolescentes de la ciudad de Lorca (Murcia). Este proyecto ha sido subvencionado por la Concejalía de Deportes del Ayuntamiento de Lorca (Murcia). En él han participado los centros de secundaria: IES Ibáñez Martín, IES Príncipe de Asturias, IES Ramón Arcas Мeca, IES Ros Giner, IES San Juan Bosco, Colegio Madres Mercedarias y Colegio San Francisco Asís. 


\section{1.- INTRODUCCIÓN.}

La inactividad física de las sociedades desarrolladas actuales constituye un factor de riesgo de enfermedades que generan grandes preocupaciones, como son las enfermedades cardiovasculares, la obesidad o la diabetes tipo II (Physical Activity Guidelines Advisory Committee, 2008; Sallis y Owen, 1999). Por este motivo, responsables y expertos de distintos países en materia de salud consideran un objetivo prioritario de salud pública la promoción de la actividad física, principalmente en los sectores más jóvenes de población, pues se encuentran en una etapa fundamental para la adquisición de hábitos de vida saludables y activos (Welk, Eisenmann y Dollman, 2006).

Dentro de este marco, preocupa el tiempo que niños y adolescentes dedican a los videojuegos, ya que el uso de estas tecnologías se considera una conducta sedentaria que compite con el ocio activo y que puede influir en una menor práctica física (Janz y Mahoney, 1997; Motl, McAuley, Birnbaum y Lytle, 2006), aunque se trata de una hipótesis controvertida, puesto que otros trabajos indican que el uso de medios tecnológicos y la actividad física no son dos caras de una misma moneda, sino conductas que pueden coexistir (Biddle, Gorely, Marshall, Murdey y Cameron, 2003). Incluso otras investigaciones indican que los niños y adolescentes más activos son los que dedican más tiempo a los videojuegos (Marshall, Biddle, Sallis, McKenzie y Conway, 2002; Martín, 2007).

De cualquier modo, y al margen de la controversia señalada, en los últimos años la concepción de los videojuegos asociada al ocio pasivo $\mathrm{y}$ al sedentarismo ha sufrido un giro radical con la aparición en el mercado de una nueva generación de videojuegos como el WiiSport para la videoconsola Wii o el Eye Toy Play Sport para la videoconsola PlayStation2, los cuales implican actividad física y han sido denominados videojuegos activos (Chin A Paw, Jacobs, Vaessen, Titze y van Mechelen, 2008; Pate, 2008).

En una sociedad inmersa en las tecnologías y con elevados índices de sedentarismo, parece que los videojuegos activos pueden representar una alternativa de práctica física, siendo parte de la solución en vez de parte del problema (Hillier, 2008). Sin embargo, aún no sabemos si el fenómeno de los videojuegos activos constituye un fenómeno social a ser tenido en cuenta como alternativa de práctica física, pues se desconoce el nivel de uso de videojuegos activos por parte de los adolescentes y si este uso compite con el tiempo dedicado a los videojuegos sedentarios o con la práctica de actividad física convencional (Beltrán, Valencia y Molina, en prensa).

Por estas razones, el presente trabajo, llevado a cabo con una muestra de adolescentes de secundaria, aborda los siguientes objetivos:

1. Conocer el tiempo que los adolescentes de la muestra dedican a la práctica de actividad física de intensidad moderadavigorosa (AFMV), a videojuegos convencionales de carácter sedentario y a videojuegos activos, y analizar si existen diferencias en función del género.

2. Estudiar las posibles relaciones entre los videojuegos activos, los videojuegos convencionales y la AFMV en el grupo de hombres y de mujeres.

\section{2.- METODO.}

\section{1.- Muestra.}

La muestra de este estudio quedó constituida por los adolescentes de $4^{\circ}$ de ESO de los 7 centros de secundaria de la ciudad de Lorca (Murcia). El $n$ de la muestra ascendió a 570 personas, de los cuales 281 fueron hombres (49,3\%) y 289 mujeres $(50,7 \%)$. Se eligió este curso por representar a los adolescentes de mayor edad dentro del sistema educativo obligatorio, en el que están representados todos los sectores de población.

\section{2.- Instrumento.}

El instrumento utilizado fue el Cuestionario de actividad física y medios tecnológicos. Se trata de un cuestionario de elaboración propia cuyo diseño se basó en la estructura del International Physical Activity Questionnaire (IPAQ), un cuestionario internacional de medida de la actividad física que cumple con condiciones adecuadas de fiabilidad y validez para adolescentes mayores de 14 años (Pinto, Correa y Pinto, 2005).

En líneas generales, se trata de un instrumento de recuerdo de las actividades de la semana anterior que recopila información sobre el tiempo dedicado a la práctica de actividad física (ligera, moderada y vigorosa) y el uso de diversos medios tecnológicos, entre los que destacan los videojuegos activos y los videojuegos convencionales. 
Tabla 1.- Diferencias en tiempo dedicado a la actividad física moderada-vigorosa según género.

\begin{tabular}{ccccccc}
\hline Sexo & Media (min./semana) & $\boldsymbol{D T}$ & Media (h/sem.) & $\boldsymbol{D T}$ & $\boldsymbol{t}$ & $\boldsymbol{p}$ \\
\hline Hombre & 508,71 & 349,63 & 8,47 & 5,82 & \multirow{2}{*}{11,32} & $<0,001$ \\
Mujer & 228,80 & 225,24 & 3,81 & 3,75 & & \\
\hline
\end{tabular}

\section{3.- Procedimiento y criterios éticos.}

En primer lugar se contactó con los directores de los distintos centros educativos de Lorca (Murcia) que imparten enseñanza en $4^{\circ}$ de ESO, para solicitar su permiso y colaboración en el estudio. Posteriormente, se hizo llegar a los padres/tutores un consentimiento informado, en el que se daban a conocer los objetivos y procedimiento del estudio, de modo que pudieran autorizar adecuadamente la participación de los adolescentes. Ninguno de los padres/tutores se negó a tal participación.

Los cuestionarios se administraron en las instalaciones del centro durante los meses de convencionales y la práctica de AFMV (franja de intensidad de especial interés desde la perspectiva de salud pública).

Los análisis estadísticos se realizaron con la ayuda del software SPSS.19.

\section{3.- RESULTADOS.}

En los siguientes apartados se presentan los principales resultados de este trabajo. Destacar que en las tablas 1,2 y 3 se expresan las medias de tiempo dedicado a AFMV, videojuegos convencionales y videojuegos activos en minutos por semana y en horas por semana para una mejor comprensión. También se añaden las respectivas desviaciones típicas (DT) de los

Tabla 2.- Diferencias en tiempo dedicado a videojuegos según género.

\begin{tabular}{ccccccc}
\hline Sexo & Media (min./semana) & DT & Media (horas/semana) & DT & $\boldsymbol{t}$ & $\boldsymbol{p}$ \\
\hline Hombre & 316,99 & 400,88 & 5,28 & 6,68 & \multirow{2}{*}{7,55} & $<0,001$ \\
Mujer & 102,76 & 258,34 & 1,71 & 4,30 & & \\
\hline
\end{tabular}

abril y mayo de 2010. El día de la administración del cuestionario se informó a los sujetos de los objetivos y pretensiones del estudio. También se recordó que el cuestionario era anónimo y la participación libre y voluntaria. Igualmente, debe destacarse que este estudio cuenta con la aprobación del Comité Ético de Experimentación de la Universidad de Sevilla, que avala el cumplimiento en este proyecto de los criterios éticos de investigación exigidos según la legislación vigente en España y la Unión Europea.

\section{4.- Análisis de datos.}

Para conocer si existían diferencias significativas en el tiempo medio empleado en AFMV, videojuegos convencionales $\mathrm{o}$ videojuegos activos en función del género, se realizaron pruebas t para muestras independientes.

Previamente, se realizaron las pertinentes pruebas de Levene para comprobar la homogeneidad de las varianzas.

También se realizaron correlaciones lineales simples para comprobar si existía algún tipo de relación entre el tiempo dedicado a los videojuegos activos, los videojuegos valores medios.

\section{Nivel de AFMV según género.}

Como puede observarse en la tabla 1, los hombres dedicaron más tiempo a la práctica de AFMV que las mujeres. Estas diferencias fueron significativas, $\mathrm{t}_{(476,18)}=11,32, \mathrm{p}<0,001$ (tabla 1 ).

\section{Tiempo dedicado a videojuegos según género.}

Si observamos la tabla 2 los análisis estadísticos indican que los hombres usaron mayor tiempo los videojuegos convencionales que las mujeres, siendo significativa esta diferencia, $\mathrm{t}_{(476,81)}=7,55$, $\mathrm{p}<0,001$ (tabla 2)

\section{Uso de videojuegos activos según género.}

El tiempo medio dedicado a los videojuegos activos fue muy bajo debido a que se trata de una tecnología mucho menos extendida entre los adolescentes.

De todos modos, puede observarse como su patrón de uso respecto al género [tabla 3] es similar al de los videojuegos convencionales, ya que son los hombres los que dedican significativamente más tiempo a videojuegos activos, $\mathrm{t}_{(367,09)}=2,39, \mathrm{p}<0,05$. 
Relación entre videojuegos activos, videojuegos. convencionales y AFMV.

Los análisis de correlación indican que, en el caso de las mujeres, la práctica de AFMV y el uso de videojuegos convencionales y activos representan conductas totalmente independientes. Sin embargo, en el grupo de hombres se observa que el uso de videojuegos activos está relacionado positivamente con el uso de videojuegos convencionales y, en menor medida, con la práctica de AFMV [figura 1]. literatura. Los resultados con la presente muestra llevan a pensar que el uso de videojuegos activos está poco extendido en los adolescentes y es muy bajo. Por lo tanto, a día de hoy, los videojuegos activos no suponen una alternativa de interés para la práctica y promoción de la actividad física. Quizá pueda existir un mayor uso en otros grupos de población de mayor edad o dentro de unos años, cuando la expansión social de estas tecnologías sea mayor, pero de cualquier modo, serán necesarias futuras investigaciones para arrojar luz sobre estas cuestiones.

Tabla 3.- Diferencias en tiempo dedicado a videojuegos activos según género

\begin{tabular}{ccccccc}
\hline Sexo & Media (min./semana) & D & Media (horas/semana) & DT & $\boldsymbol{t}$ & $\boldsymbol{p}$ \\
\hline Hombre & 9,29 & 41,07 & 0,15 & 0,68 & \multirow{2}{*}{2,39} & $<0,05$ \\
Mujer & 2,97 & 16,60 & 0,04 & 0,27 & & \\
\hline
\end{tabular}

\section{4.- DISCUSION Y CONCLUSIONES.}

En este estudio se observa que la práctica de actividad física es superior en chicos que en chicas. Otros estudios con adolescentes avalan este resultado (Sallis y Owen, 1999; Peiró, Devís, Beltrán y Fox, 2008, Román, Serra, Ribas, Pérez y Aranceta, 2006) y señalan la necesidad de considerar a las chicas un grupo social de atención preferente en las estrategias de promoción de la actividad física.

En función de los valores medios de AFMV, podemos afirmar que la muestra del presente trabajo es bastante activa. No obstante, debemos remarcar que las chicas no cumplen con la recomendación de los expertos para niños y adolescentes de realizar 60 o más minutos diarios de AFMV (Physical Activity Guidelines Advisory Committee, 2008). El uso de videojuegos convencionales por parte de los adolescentes varones es superior al de las chicas, dato que coincide con los resultados de la literatura (Bercedo et al., 2005; Vanderwater, Shim y Caplovitz, 2004; Colwell y Kato, 2005). También es similar el tiempo empleado en videojuegos por parte de los chicos de la muestra en comparación con otros trabajos que hablan de medias de uso de videojuegos entre los 30 minutos diarios y una 1 hora (Garitaonandía, Fernández y Oleaga, 2004, Bercedo et al., 2005; Vanderwater et al., 2004), aunque el tiempo empleado por las chicas de este estudio es menor a los valores que contemplan estos estudios.

Un dato muy novedoso de este trabajo es el tiempo dedicado a videojuegos activos, no habiéndose encontrado precedentes en la
En lo que concierne a las relaciones entre los videojuegos activos, los videojuegos convencionales y la práctica de AFMV, destaca que en ninguno de los dos grupos existe relación entre AFMV y videojuegos convencionales. Por lo tanto, no podemos afirmar que este tipo de videojuegos contribuya a la problemática del sedentarismo. En este sentido, como ya se ha apuntado en la introducción de este trabajo, cabe destacar que estudios previos ya han señalado que el uso de los videojuegos puedes coexistir perfectamente con la práctica física (Biddle et al., 2003) y que incluso los niños y adolescente más activos son aquellos que dedican más tiempo a los videojuegos sedentarios (Marshall et al., 2002, Martín, 2007).

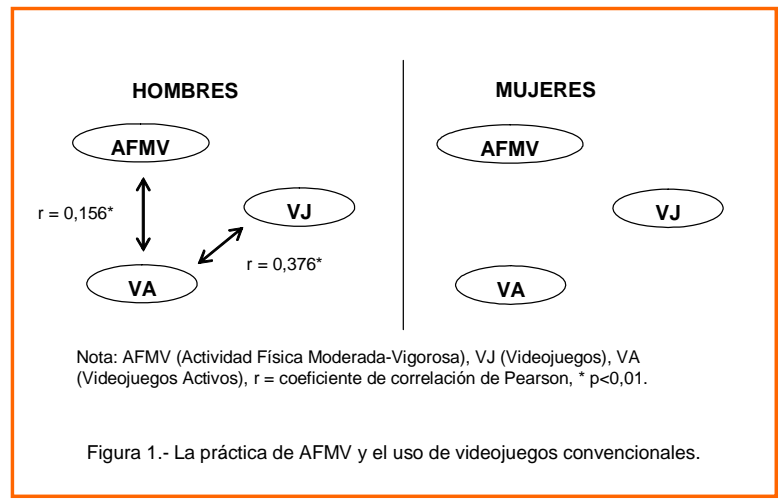

Otro dato a destacar es que en el grupo de hombres, los que más utilizan videojuegos activos son aquellos que más juegan a los videojuegos convencionales y practican más AFMV. Por lo tanto, los videojuegos activos, ni restan tiempo a los videojuegos convencionales de carácter sedentario, aspecto que sería deseable desde el punto de vista de la prevención del sedentarismo, ni compiten con la práctica de 
actividad física convencional, aspecto que sería especialmente preocupante.

Mientras se prevé en un futuro próximo una mayor sofisticación y extensión del fenómeno de los videojuegos activos en nuestra sociedad, la información aportada en este trabajo puede ser de interés para todas aquellas personas preocupadas por el sedentarismo de los adolescentes.

\section{5.- REFERENCIAS BIBLIOGRÁFICAS.}

Beltrán, V.J., Valencia, A. \& Molina, J.P. Los videojuegos activos y la salud de los jóvenes: Revisión de la investigación. Revista Internacional de Medicina y Ciencias de la Actividad Física y el Deporte. En prensa.

Bercedo, A., Redondo, C., Pelayo, R., Gómez, Z., Hernández, M. \& Cadenas, N. (2005). Consumo de los medios de comunicación en la adolescencia. Anales de Pediatría, 63(6), 516525.

Biddle, S.J., Gorely, T., Marshall, S.J., Murdey, I. \& Cameron, N. (2003). Physical activity and sedentary behaviours in youth: issues and controversies. The Journal of The Royal Society for the Promotion of Health, 124(1), 29-33.

Chin A Paw, M. J. M., Jacobs, W. M., Vaessen, E. P. G., Titze, S. \& van Mechelen, W. (2008). The motivation of children to play an active video game. Journal of Science and Medicine in Sport, 11(2), 163-166.

Colwell, J. \& Kato, M. (2005). Video game play in British and Japanese adolescents. Simulation and Gaming, 36(4), 518-530.

Garitaonandía, C., Fernández, E. \& Oleaga, J.A. (2004). Las tecnologías de la información y de la comunicación y su uso por los niños y los adolescentes. Doxa, 3, 45-64.

Hillier, A. (2008). Childhood overweight and the built environment: Making technology part of the solution rather than part of the problem. The Annals of the American Academy of Political and Social Science, 615(1), 56-82.

Janz, K.F. \& Mahoney, L.T. (1997). Maturation, gender, and video game playing are related to physical activity intensity in adolescents: The Muscatine Study. Pediatric Exercise Science, 9(4), 353-363.

Marshall, S.J., Biddle, S.J.H., Sallis, J.F., McKenzie, T.L. \& Conway, T.L. (2002). Clustering of sedentary behaviors and physical activity among youth: a cross-national study. Pediatric Exercise Science, 14(4), 401-417.

Martín, M. (2007). Nivel de actividad física y de sedentarismo y su relación con conductas alimentarias en adolescentes españoles. Tesis Doctoral. Granada: Universidad de Granada.
Motl, R. W., McAuley, E., Birnbaum, A. S. \& Lytle, L. A. (2006). Naturally occurring changes in time spent watching television are inversely related to frequency of physical activity during early adolescence. Journal of Adolescence, 29(1), 1932.

Pate, R. R. (2008). Physically active video gaming an effective strategy for obesity prevention? Archives of Pediatrics \& Adolescent Medicine, 162(9), 895-896.

Peiró, C., Devís, J., Beltrán, V.J. \& Fox, K.R. (2008). Variability of Spanish adolescents' physical activity patterns by seasonality, day of the week and demographic factors. European Journal of Sport Sciences, 8(3), 163-171.

Physical Activity Guidelines Advisory Committee. (2008). Physical Activity Guidelines Advisory Committee Report, 2008. Washington: Department of Health and Human Services.

Pinto, D., Correa \& C., Pinto, J.E.R. (2005). Reproducibility and validity of the International Physical Activity Questionnaire in adolescents. Revista Brasileira de Medicina do Esporte, 11(2), 147e-154e.

Román, B., Serra, L., Ribas, L. \& Pérez, C. \& Aranceta, J. (2006). Actividad física en la población infantil y juvenil española en el tiempo libre. Estudio enKid (1998-2000). Apunts. Medicina de l'Esport, 151, 86-94.

Sallis, J.F. \& Owen, N. (1999). Physical activity and behavioral medicine. Thousand Oaks: Sage Publications.

Vandewater, E. A., Shim, M. \& Caplovitz, A. G. (2004). Linking obesity and activity level with children's television and video game use. Journal of Adolescence, 27(1), 71-85.

Welk, G.J., Eisenmann, J.C., \& Dollman, J. (2006). Health-related physcal activity in children and adolescents: a bio-behavioral perspective. In D. Kirk, M. O’Sullivan, \& D. Macdonald (Eds.), The Handbook of Physical Education (pp. 666684). London: Sage.

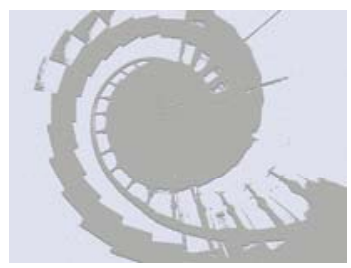

